\begin{tabular}{|c|c|}
\hline Title & The Significance of the A queous Ice Phase Transformation during Controlled Rate Cooling of Biological Specimens \\
\hline Author(s) & Rowe, Arthur W. \\
\hline Citation & Cellular Injury and Resistance in Freezing Organisms: proceedings, 2, 21-31 \\
\hline Issue Date & 1967 \\
\hline Doc URL & http:/hdl .handle.net/2115/20406 \\
\hline Type & bulletin (article) \\
\hline Note & $\begin{array}{l}\text { International Conference on Low Temperature Science. I. Conference on Physics of Snow and Ice, II. Conference on } \\
\text { Cry obiology. (A ugust, 14 19, 1966, Sapporo, Japan) }\end{array}$ \\
\hline File Information & 1_p21-31.pdf \\
\hline
\end{tabular}

Instructions for use 


\title{
The Significance of the Aqueous-Ice Phase \\ Transformation during Controlled Rate Cooling of Biological Specimens*
}

\author{
Arthur W. Rowe \\ The New York Blood Center, New York, N.Y., U.S.A.
}

\begin{abstract}
The controlled cooling of biological materials to subzero temperatures involves passage through several phases: the liquid aqueous phase, the aqueous to ice transformation or heat of fusion phase, and the solid ice phase. Cooling rate in the liquid phase prior to entering the heat of fusiontransformation phase appears to be of least significance in most biological systems. The time involved for liquid to solid (aqueous to ice) phase transformation and concomitant latent heat of fusion removal appears to be of considerable biological significance. Decreasing biological viability is obtained as the duration of the specimen in the heat of fusion phase is prolonged, presumably due to prolonged exposure to dehydration effects of water-ice crystals and increased concentration of solutes. Rate of heat removal in the post-heat of fusion solid state is also important in maintaining viability of the biological specimen. Experimental biological evidence supports these contentions and provides a basis upon which to formulate and verify some basic concepts of freezing injury.
\end{abstract}

\section{Introduction}

Cellular systems survive best at low temperatures where conditions are adjusted so as to minimize damage that might occur to their macromolecular and structural integrity. To circumvent the otherwise lethal effects of freezing and thawing, one must understand the behavior of the system; that is, the physical phenomena of phase equilibria and thermal energy removal and input, as well as the chemical phenomena such as composition, solute effects on crystallization, solution structure, and, last but not least, the function of cryoprotective additives (CPA's) in relationship to maintenance of viability (Doebbler et al., 1966; Rowe, 1966). It is the cryobiologist who is attempting to interpret, control, and apply the phenomena of freezing and thawing at an interdisciplinary level.

With any particular cell type a quantitative relationship may be unique between cooling rate and injury, yet qualitative relationships may exist. Current beliefs about the relationship between cooling rate and level of viability in cells (Doebbler and Cowley, 1964; Mazur, 1965) is diagrammatically presented in Fig. 1. Very slow cooling rates (area $A$ ), on the order of fractions of degrees per minute, allow the formation of large ice crystals, concentration of solutes (i.e., salts) in the surrounding medium, dehydration or removal of intracellular water. These phenomena make the possibility of intracellular ice damage rather unlikely. Damage, then, is principally due to dehydration and concomitant solute concentration with resulting irreversible disruption of ordered

* These studies were supported in part by research funds from Union Carbide Corporation, Linde Division, and the National Heart Institute of the National Institutes of Health. 


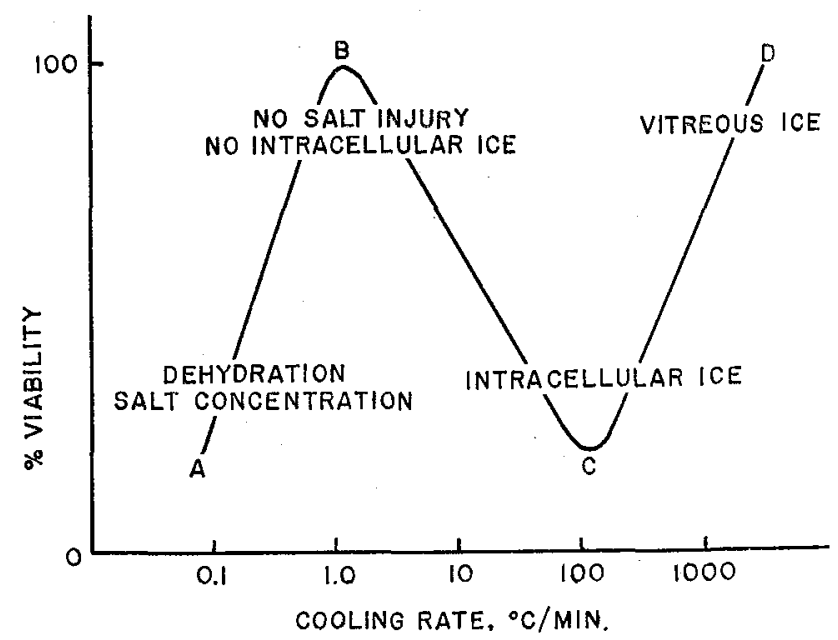

Fig. 1. Diagrammatic representation of some prevailing theories of relationship between cooling rate and level of viability in cells

molecular structure, principally within cellular membranes. Somewhat more rapid cooling (area B), on the order of one to 10 degrees a minute, limits the time of exposure to the concentrated solutes in the medium with reduction in damage to susceptible molecular constituents. Intracellular water is correspondingly lost to a degree where it is also unlikely that formation of damaging intracellular ice crystals occurs. Greatest viability is therefore retained in this region of cooling rates, resulting in a cooling regimen which is expanded upon in Fig. 2 and which will be discussed in greater detail later.

With rapid cooling (area C), injury to the cell by concentrated solutes in the medium

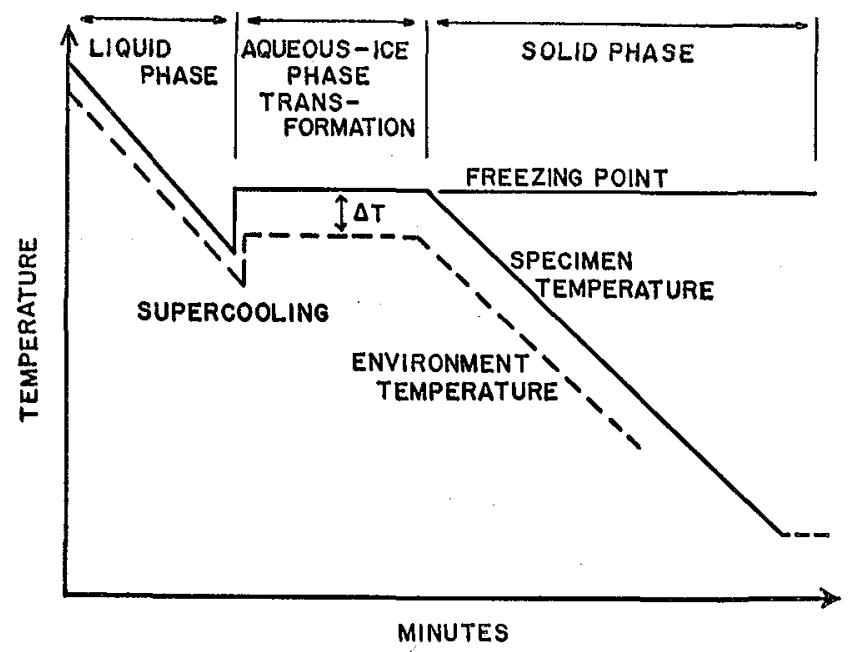

Fig. 2. Typical curve of slow controlled rate cooling indicating the three principal areas of 1) liquid phase, 2) aqueous-ice phase transformation, and 3) solid phase. The specimen temperature is represented by the solid line while the lower dotted line represents the temperature of the surrounding environment which can be varied to effect the desired cooling rates 
is largely avoided because of short exposure time. At this rapid rate, however, water may not escape from the cell rapidly enough and result in intracellular ice nucleation with resulting damage (Asahina, 1965). It is entirely possible to freeze so rapidly (area D) that the size of ice crystals may be of such small size as to have a negligible damaging effect, or even a semicrystalline or vitreous state may occur. At best, this curve representing prevailing hypotheses holds only for the information currently available, yet must remain flexible enough for modification as new knowledge is attained. No single event can serve to explain the increasingly complex phenomena that occur during freezing and thawing of biologicals. While generalizations may be made, each system is unique in itself.

To retain viability after freezing and thawing, most nucleated mammalian cells have to be frozen at a controlled rate in the presence of a cryoprotective additive (Rowe, 1966). The nonnucleated human erythrocytes, however, are capable of withstanding a far broader range of freezing conditions in the presence of a greater variety of protective compounds (Mazur, 1966).

\section{A Slow Cooling Regimen}

This discussion will be limited largely to the slow controlled rate cooling regimen (area B of Fig. 1) which has been so successful for preservation of viability yet fraught with limited understanding of the phenomena taking place. Figure 2 is a stylized freezing regimen illustrating some of the major component parts, the shape of which is actually a summation of transient heat transfer (Rowe, 1966). In the presence of cryoprotective additives of suitable concentration (e.g., glycerol, dimethyl-sulfoxide), it is through control of this regimen that the least damage to nucleated cells after freezing and thawing has resulted. The major regions of this diagrammatic representation of a slow cooling regimen are 1) the liquid phase, 2) aqueous-ice phase transformation area, and 3) solid phase.

Liquid phase. Hypothermia, or cooling of the liquid phase from ambient to the freezing point, is probably of the least overall significance. Various biochemical reaction rates markedly decrease and may result in occasional, transient or reversible damage (Mazur, 1966). With but few exceptions cooling in this region appears to have little permanent effect on the cells. Cells such as spermatozoa (Smith, 1961, p. 440) are probably the most notable exception, undergoing a profound loss of viability due to "hypothermal cold shock" during rapid cooling to the freezing point. Scattered reports in the literature do emphasize the importance of the liquid state time temperature relationships (Rinfret, 1966). The overwhelming majority of cellular systems, however, seem to tolerate this cooling regimen well. This liquid phase also encompasses the supercooling state where the aqueous solute system is at a temperature below its crystallization point. Very little has been studied about the biological effects in this specific region, but most data suggest that it is well tolerated in the systems not affected by thermal shock.

Aqueous-ice phase transformation. At the freezing point of this system in question the phase transition area, or isothermal plateau, occurs; that is to say, the water in liquid milieu releases its latent heat of fusion and transforms into the ordered solid ice phase. This water-to-ice transformation phase is represented by a plateau in a temperaturetime relationship. With extremely rapid rates of cooling this transition phase is often 
indistinguishable from the cooling curve as a whole (Rinfret, 1966). It is slower rates, on the order of degrees per minute, that result in a pronounced heat of fusion phase. As the bulk of the system or effective path of transient heat transfer increases, the significance of the phase becomes of considerable consequence (Rowe and Rinfret, 1962), as will be shown by data in a later section of the paper.

Solid phase. After completion of the phase change in the area of the freezing point, the solid phase then continues to cool. Because of different thermal properties (thermal conductivity, diffusivity, etc.) for solid and liquid states, the temperature drop of the solid phase can proceed at a rate faster than was possible with the liquid phase. The presence of solutes, notably salts, still results in the presence of some unfrozen solvent until the eutectic point (or region) is passed. In the case of a pure sodium chloride solution this solute continues to concentrate until it reaches about $24 \%$ by weight at the eutectic temperature $-21.6^{\circ} \mathrm{C}$. Passing the eutectic point results in the crystallization of the sodium chloride dihydrate with additional ice. Much work is still necessary to define further the eutectic of the system. Controlling the rate of cooling to below the sodium chloride eutectic of the system appears to result in the least damage to the cells. It must be appreciated that one can talk only of a theoretical eutectic range as the presence of cryoprotective additives and so many solutes in the biological system makes it virtually impossible to measure the eutectic of a specific solute and specify it as the eutectic of the system. Below this range the rate of cooling plays a minor role (Mazur, 1966).

Control of cooling rate. An empirically useful method of cooling rate control is generally tied to a particular biological system in terms of its size and thermal characteristics. Such seems to be true for low temperature preservation of most mammalian cell types as well as microorganisms. Biological specimens have often been cooled slowly by being exposed to environments of static cold gaseous atmospheres or liquids cooled by secondary refrigeration. Because the size and thermal properties of the individual specimens varied considerably, such procedures offered no control over cooling rate in various regions of the specimen, resulting in thermal gradients of different magnitudes within the specimen itself (Rinfret, 1966; Meryman, 1966).

We have observed that in a bath (e.g., dryice-alcohol) being cooled at a constant rate, the rate of cooling in the specimen itself will depend markedly upon the volume and cross sectional heat transfer zone. Under a small temperature gradient, removal of the latent heat of fusion takes much longer as the volume of the specimen increases. The constant cooling bath temperature may fall considerably below the freezing point of the specimen during this time, and once the phase transition is complete the cooling of the solid phase will be uncontrollably rapid because of the higher thermal conductivity of ice as compared with water (Meryman, 1966).

We have been controlling the rate of cooling and heat of fusion of biological specimens using the sample itself as a reference point in the system (Rowe and Rinfret, 1962). This can be accomplished using a biological freezer (Linde Division of Union Carbide Corporation, New York, N.Y.) where cold nitrogen gas is injected by a feedback mechanism in amounts sufficient to cool the specimen at the prescribed monitored rate. This is accomplished by a differential thermocouple from which signals are fed into a relay 


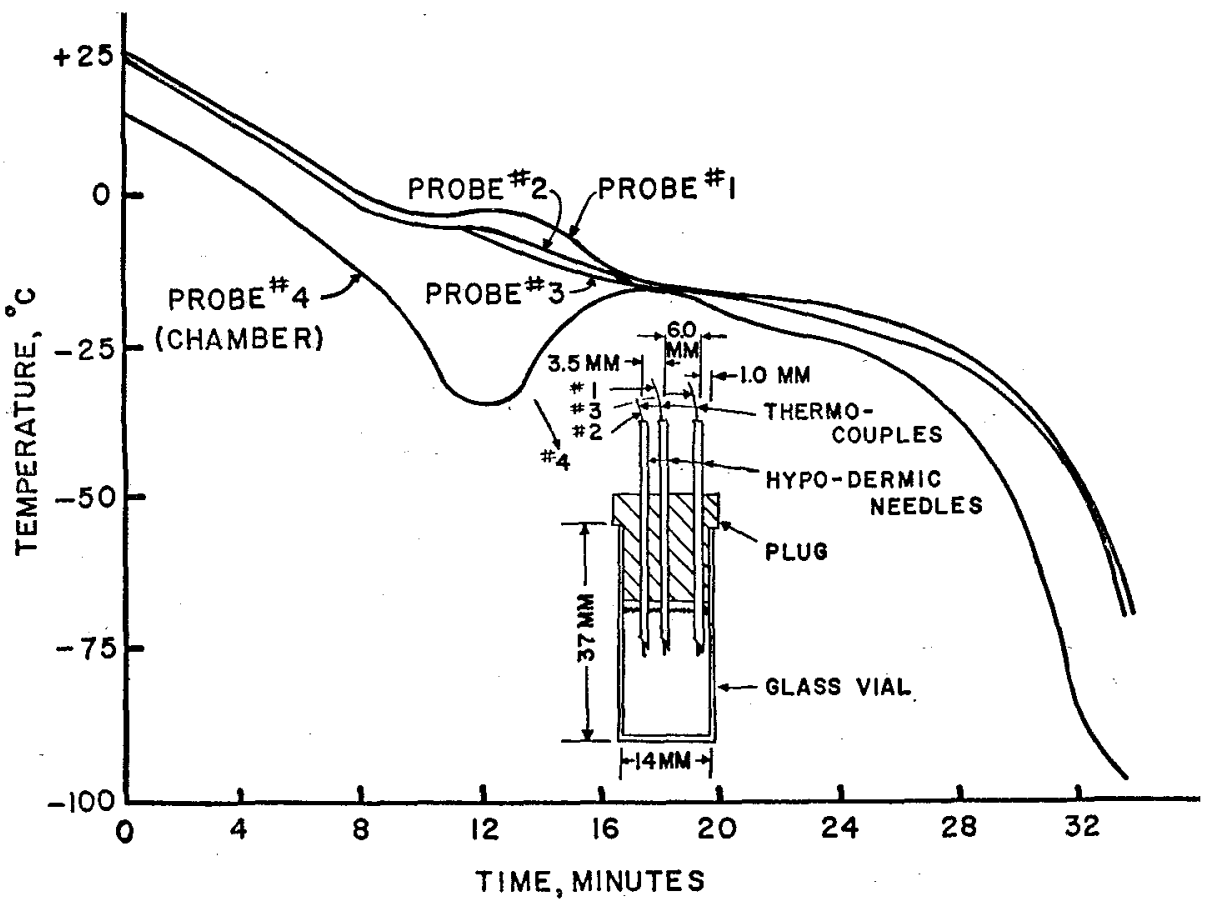

Fig. 3. Freezing curve for a solution containing $10 \%$ dimethyl-sulfoxide (DMSO). Four $\mathrm{ml}$ of solution were frozen in a glass ampule with specifically placed thermocouples using a biological freezer (Linde Division, Union Carbide Corp.)

amplifier biased by an adjustable reference circuit (Doebbler and Cowley, 1964). The higher the bias setting the greater will be the temperature difference maintained between the sample and its environment, and the faster will be the cooling rate.

Cooling of biological specimen by the differential thermocouple cold gas environment is seen in Fig. 3. Thermocouples are positioned at various sites, namely the geometric center (where the control thermocouple is, also), the point midway between the center and outer edge, and the outer periphery. This was done to measure and determine where thermal gradients occur. With this cooling system the chamber or environment temperature is about 10 degrees colder than the specimen, producing a cooling rate in the liquid phase of the specimen of 1-2 degrees per minute. Upon reaching the heat of fusion phase, the $\Delta T$ or temperature differential ( $c f$. Fig. 2) between the chamber's cold environment and the specimen had to be increased considerably to compensate for the plateau phase where heat of fusion was being liberated during the aqueous-to-ice phase transformation. At the termination of that phase it is necessary to decrease the temperature difference between the environment and specimen to minimize temperature gradients in the sample. It should be noted that the greatest thermal gradients in the sample occur at the end of the heat of fusion phase and it is here that tremendous gradients have been recorded when constant rate cooling baths were used. The object is to keep these gradients to a minimum because a cooling rate is impossible to define when cells experience different thermal regimens depending upon their location in the 
specimen container. The best we can do is approach an overall average rate with minimal deviation.

An increase in volume of the specimen container will not make any difference if the geometry of the specimen is kept constant or uniform for heat transfer purposes. This becomes most obvious when attempting to cool organs of increased volume and of different geometry. By empirical observation we have been able to achieve a rather uniform cooling curve by placing the specimen in cold atmosphere at $-140^{\circ} \mathrm{C}$. A sizable thermal gradient does occur in the sample, however, as noted by a marked difference in temperature between the thermocouple closest to the outer edge and the two thermocouples in the inner section and geometric center of the specimen container.

Warming rate. It is generally agreed that warming is of importance and for highest viability (Mazur, 1966) needs only to be rapid to prevent ice crystal growth and concomitant cellular damage with prolonged exposure. There has been little study of this aspect of the cooling and warming cycle and it may share more importance than is ordinarily attributed to it. In Fig. 4 we have placed a frozen specimen in a warm water bath at $42^{\circ} \mathrm{C}$. The periphery of the sample warms quicker than the center, as

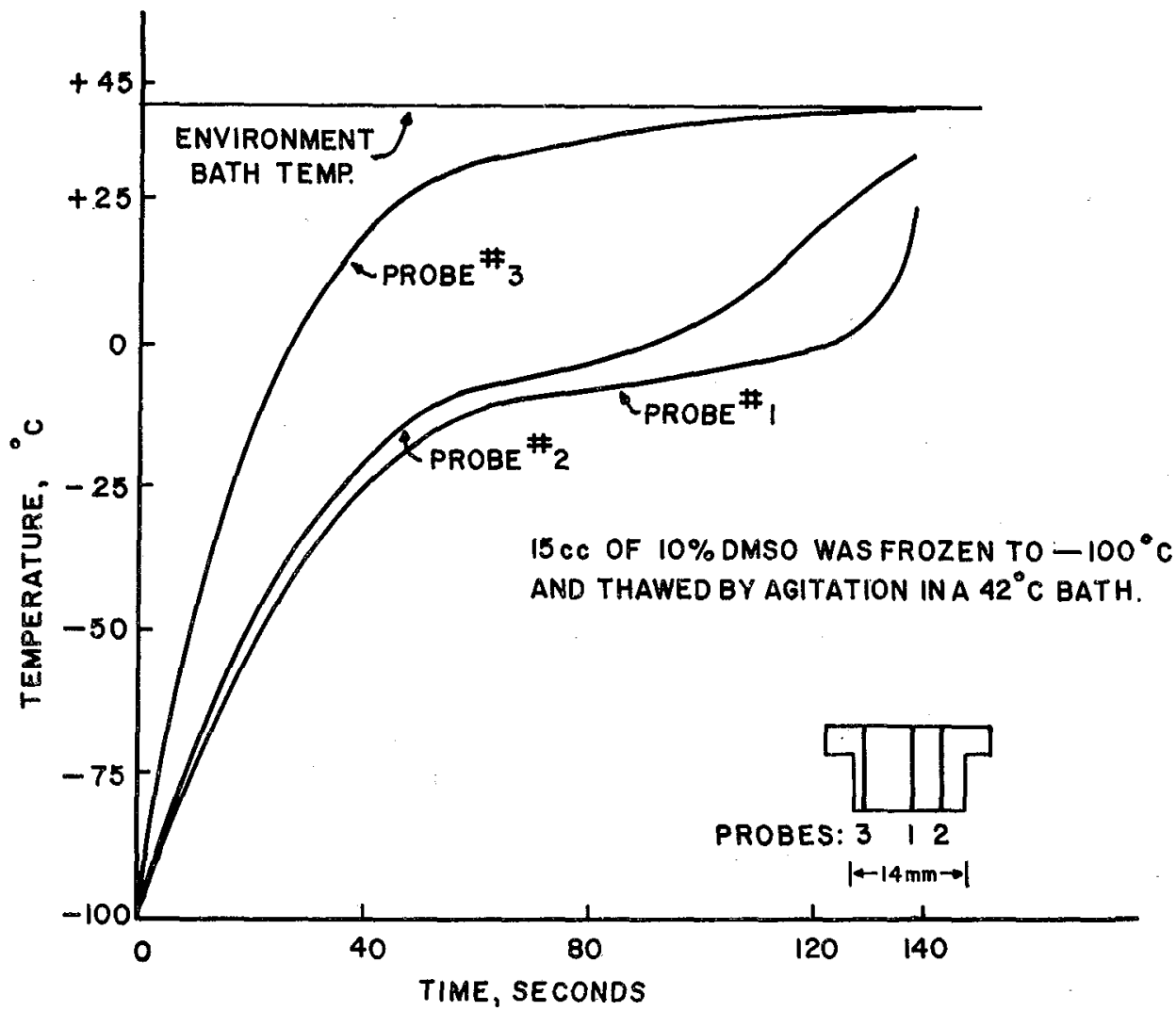

Fig. 4. Thawing curve of a tube containing $10 \%$ dimethyl-sulfoxide in plasma while gently agitating in a $42^{\circ} \mathrm{C}$ water bath. Large thermal gradients are apparent particularly the center probe 11 where the ice mass remains long after the periphery of the sample has been thawed 
expected, and tremendous temperature gradients are observable. As the center approaches the freezing, or rather melting, point, the phase plateau is again observed over a finite time span during which biological changes could take place.

Specimen geometry. Since cooling rate (and warming rate) can only be an average value based on effective heat transfer path, a suitably uniform geometric configuration. is necessary to obtain the best average with least deviation (Meryman, 1966). The significance of the geometry of the specimen becomes most apparent when we deviate from a cell suspension that can be in a container of any material or any size and go to organized cell structures such as one encounters in organ freezing. In the case of an organ of considerable bulk and having a large heat transfer path, such as a kidney, there is considerable difficulty in maintaining a uniform gradient throughout the organ as a whole during cooling. A circular plastic block was used to surround a kidney which was kept suspended in the center by four needles, allowing complete exposure of the organ to the cooling environment. Holes were drilled in three planes of the plastic block so that thermocouples could be inserted through the needles and situated in various areas of the organ to monitor thermal gradients. After a considerable amount of difficulty a cooling regimen was achieved where the temperature gradients in the kidney were not excessive. This is shown in Fig. 5 where four thermocouples have

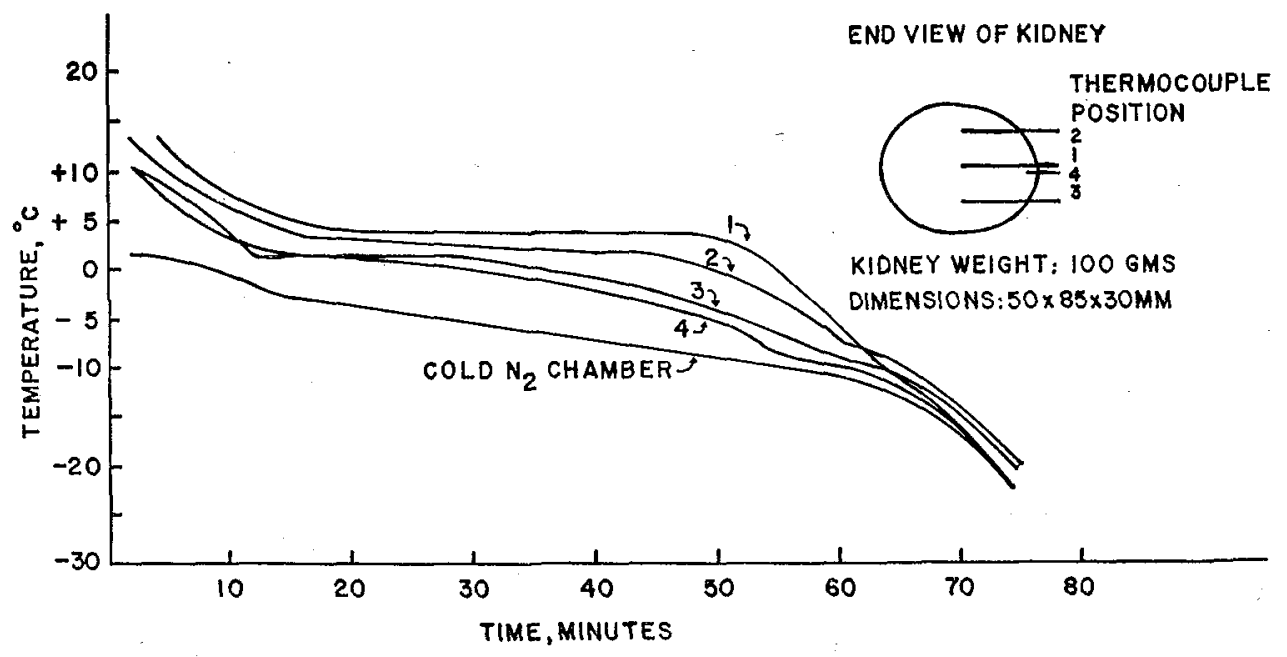

Fig. 5. Thermal profiles of a kidney (perfused with $10 \%$ DMSO) during slow controlled rate cooling using cold nitrogen gas in a biological freezer (Linde)

been positioned in different areas: the geometric center, the planes above and below the geometric center, and the periphery of the organ (see insert with end view showing thermocouple position). As noted previously, the area at the end of the phase change resulted in the largest thermal gradients in the sample. The sacrifice that was necessary to keep the thermal gradients at a minimum was to prolong the latent heat of fusion time. This prolongation of heat of fusion time has been found detrimental biologically, as will be pointed out later. Upon thawing of this large tissue mass we have been able to preserve, as far as we can tell, reasonably normal cellular morphology. We have not 
been able as yet to demonstrate any evidence of functionability.

Biological implications. The two most important zones appear to be the phase transition time and the cooling rate immediately thereafter (Rowe and Rinfret, 1962; Lewis et al., 1965; Rowe, 1966). Rowe and Rinfret (1962) were the first to emphasize the importance of these zones based on some in vitro metabolic parameters, namely cellular respiration and protein synthesis as measured by incorporation of glycine-2- $\mathrm{C}^{14}$ into acid insoluble proteins. Thermal shock to nucleated cells such as bone marrow cells has not been observed with liquid or pre-heat of fusion phase, as seen by the data in Table 1. The rate of cooling in the post-heat of fusion phase, however, was found to be significant. A slower rate, on the order of $1^{\circ} \mathrm{C}$ per minute, was found to be better biologically than rates which were faster.

Table 1. Effect of cooling rate before and after the heat of fusion transformation phase on viability of bone marrow cells

\begin{tabular}{lccc} 
& Cooling rate & $\begin{array}{c}\text { Viability* as percent of control } \\
\text { Pre-heat of fusion }\end{array}$ & 74 \\
Slow & $1^{\circ} \mathrm{C} / \mathrm{min}$ & 69 & 58 \\
Medium & $3-4^{\circ} \mathrm{C} / \mathrm{min}$ & 72 & 56 \\
Fast & $8-9^{\circ} \mathrm{C} / \mathrm{min}$ & & 45 \\
(Phase transition time-2 $\mathrm{min}$ ) & & \\
\hline
\end{tabular}

Effect of time spent in phase transformation on viability of bone marrow cells

\begin{tabular}{ccc}
\hline $\begin{array}{c}\text { Phase transition time } \\
\text { (Heat of fusion period) }\end{array}$ & \multicolumn{2}{c}{$\begin{array}{c}\text { Viability* as percent of control } \\
15 \%\end{array}$} \\
\hline $1-2$ minutes & 45 & $15 \%$ DMSO \\
$8-10$ minutes & 33 & 52 \\
$15-25$ minutes & 3 & 35 \\
$\left(\right.$ Cooling rate $\left.-1^{\circ} \mathrm{C} / \mathrm{min}\right)$ & & 2 \\
\hline
\end{tabular}

* Average viability by in vitro measurement of cellular respiration and protein synthesis using incorporation of glycine-2-C $\mathrm{C}^{14}$

(From Rówe, 1966)

The aqueous-to-ice phase transformation, isothermal, or latent heat of fusion period, is perhaps the most critical part of the cooling curve. It can be observed that the shortest time spent in the isothermal phase was better than longer times for optimum viability. Of course, the larger the bulk of the specimen the greater the importance of control of thermal uniformity becomes. It is perhaps during this isothermal phase where ice crystals are formed and dehydration of the cells occurs, with concomitant salt concentration and resulting cell membrane denaturation upon prolonged exposure, that biological damage is manifested most unless the temperature is lowered. Biological damage is diminished as the temperature of the system is lowered (Farrant, 1965), particularly below the eutectic range of the system.

These data on the biological effect of isothermal and cooling times are based on in 
vitro criteria which are especially useful because they offer a sensitive measure of the cell population as a whole and do not necessarily account for a selected thermally hyposensitive population. In the case of bone marrow, clinically oriented investigators are most obviously interested in the repopulation capacity and hence preservation of a select population of cells, i.e., stem cells. Our use of a panel of in vitro viability criteria offers a variety of screening methods capable of measuring different parameters of the cell. Animal assays, on the other hand, will indicate the repopulation potential of bone marrow but are difficult to quantitate because they tend to provide an all-or-none effect which is variable depending upon the species under study. Our results were obtained with several viability parameters, in this case respiration and incorporation of $\mathrm{C}^{14}$-glycine into protein, which correlate well with irradiated animal assays of our own and of others (Rowe and Fellig, 1963; Rowe, 1966).

In some rather elegant studies, Lewis et al. (1965) have corroborated our in vitro data on the biological significance of cooling rate and latent heat of fusion phase. They accomplished this using an in vivo irradiated animal assay under very carefully controlled conditions. Rather than look for the rather insensitive 30-day survival, they used the Till and McCullough's (1961) spleen colony count which is quicker and offers better quantitation. This assay is based on observation that hematopoietic cell recovery in irradiated mice occurs as discrete nodules or colonies of hematopoietic tissue on the spleen. These nodules are related to the numbers of viable cells injected and provide a basis for quantitative assay of repopulation potential. With this method of viability assay they found no pre-heat of fusion rate dependency but, as seen in Fig. $6 \mathrm{~A}$, were able to demonstrate a rate dependence in the post-heat of fusion phase, the slower rate being better than faster cooling rates. These studies (Lewis et al., 1962) further corroborated ours (Rowe and Rinfret, 1962; Rowe, 1966) by showing in Fig. 6B a marked dependence on the heat of fusion or aqueous-ice transformation phase. The duration spent in this isothermal region by the specimen is biologically significant, the shorter times resulting in higher viability than longer times.
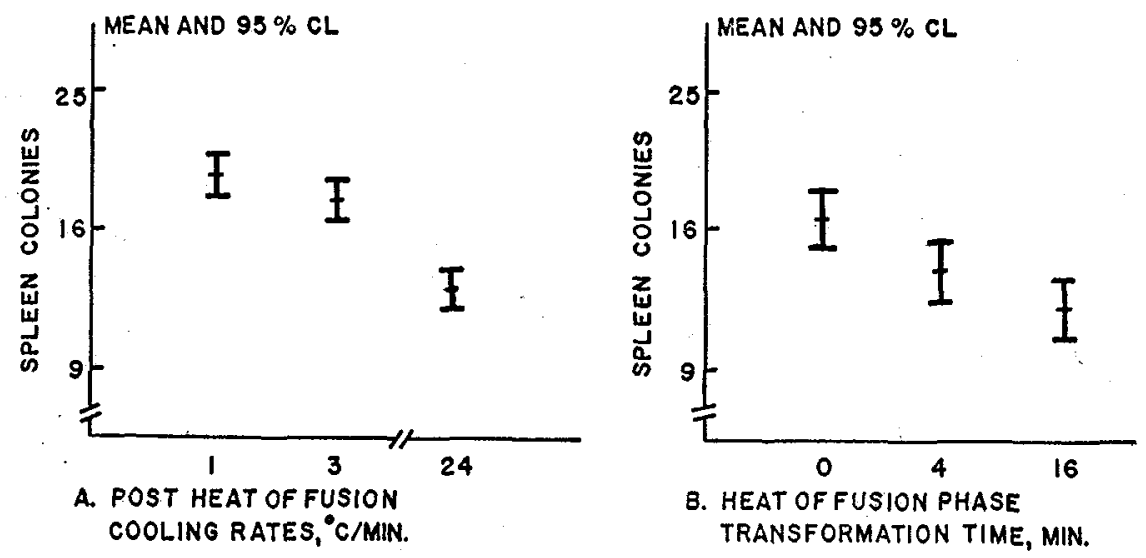

Fig. 6. Effect of cooling rate $(A)$ and latent heat of fusion transformation time (B) on viability of bone marrow based on spleen colony counting at $95 \%$ confidence limits (95\% CL). (Data by permission of Lewis et al., 1965) 
We have also carried out studies on the effect of isothermal (aqueous-ice transformation) phase duration using tissue culture of primary kidney cells of the rabbit. The broken line in Fig. 7 shows the expected normal growth of cortical kidney cells. The

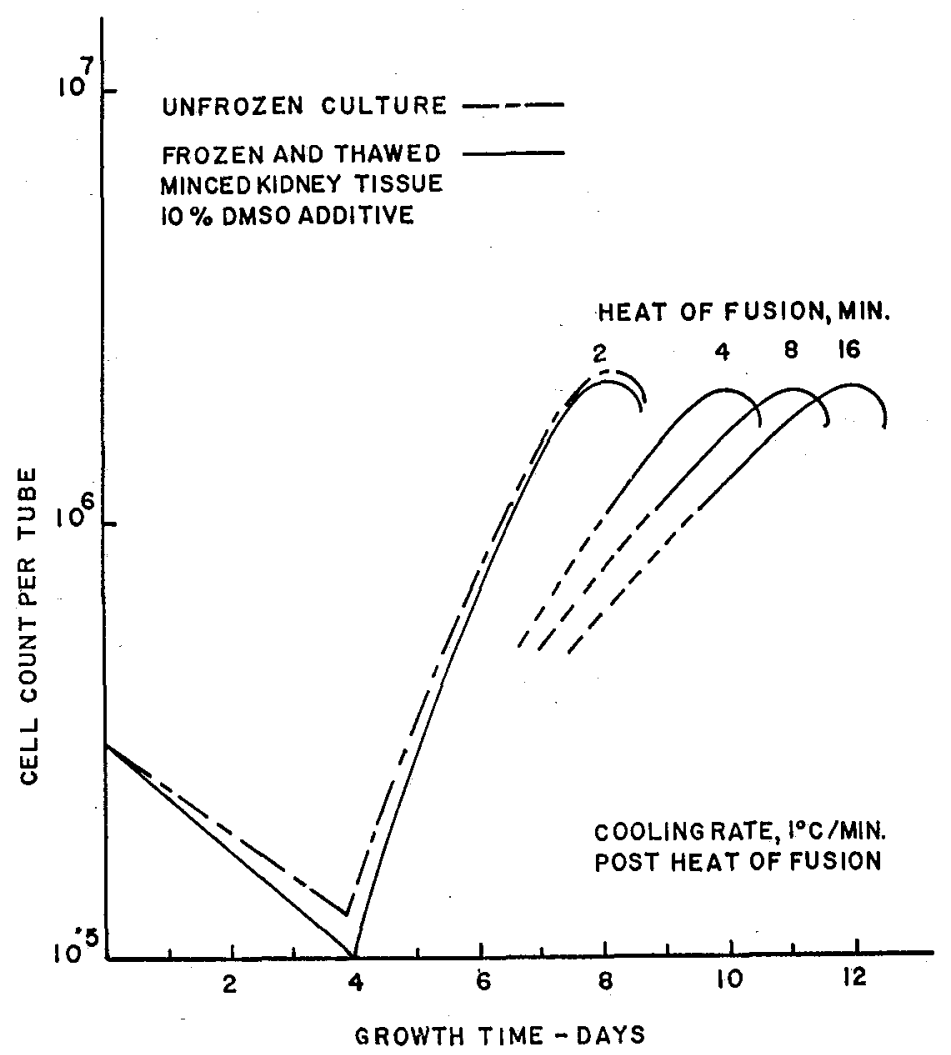

Fig. 7. Effect of freezing and latent heat of fusion on growth rate of rabbit kidney cortical tissue. The kidney cells were frozen as minced tissue in a serum-nutrient medium containing $10 \%$ dimethyl-sulfoxide using $14 \mathrm{~mm}$ diameter aluminum tubes. A controlled cooling rate of $1^{\circ} \mathrm{C}$ per minute before and after the heat of fusion phase was maintained

cells are inoculated at a specific level, the count normally decreases in the first few days, and cell growth then commences until a peak is reached in seven to eight days. The solid line represents the growth of frozen and thawed kidney tissue using a $10 \%$ DMSO additive. Using the same inoculation level of viable cells based on trypan blue vital staining, the time that the sample reached peak or maximum cell growth in culture tubes was observed. As the heat of fusion time is increased in the cooling regimen of one degree per minute there is a slight effect on the trypan blue vital staining, but there is a marked delay in the time the cells take to reach peak growth, again illustrating the biological viability dependence on thermal regimen and duration of latent heat of fusion.

\section{Conclusion}

In conclusion, I should like to emphasize the importance of controlling the rate of 
cooling and the duration of the aqueous-ice phase transformation (heat of fusion, isothermal, etc.) time. It is preferable to maintain the specimen of a suitable geometric configuration where unusual thermal gradients are kept to a minimum. Our biological data and those of others support the contention that the time the specimen spends in the latent heat of fusion should not be prolonged, and once the isothermal range is passed the cooling rate should be kept at a uniform, slow rate to some temperature below the eutectic range, after which the cooling rate may be increased. The dependence upon and biological importance of maintaining a controlled cooling regimen warrants further intensive study on sub-cellular and molecular phenomena taking place in this narrow temperature range where the aqueous-ice phase transformation occurs and the cooling immediately thereafter.

\section{References}

AsAHINA, E. 1965 Freezing process and injury in isolated animal cells. Fed. Proc., 24, Suppl. 15, S-183-187.

Doebbler, G. F., Rowe, A. W. and Rinfret, A. P. 1966 Freezing of mammalian blood and its constituents. In Cryobiology (H. T. MERYMAN, ed.), Academic Press, New York, 407-450.

Doebbler, G. F, and Cowley, C. W. 1964 Cryobiology. Interntl. Science and Technology, 58-70.

FARRANT, J. 1965 Mechanism of cell damage during freezing and thawing and its prevention. Nature, 205, 1284-1287.

Lewis, J. P., Passovoy, M. and Trobaugh, F. E., JR. 1965 Studies on the effect of controlled rate cooling on marrow viability. Proc. 10th Congr. Int. Soc. Blood Transf., Stockholm, 1964, Karger, Basel/New York, Bibl. Haemat., 23, 656-661.

Mazur, P. 1965 Causes of injury in frozen and thawed cells. Fed.Proc., 24, Suppl. 15, S-175-182.

MAZUR, P. 1966 Theoretical and experimental effects of cooling and warming velocity on the survival of frozen and thawed cells. Cryobiology, 2, 181-192.

MERYMAN, H. T. 1966 The interpretation of freezing rates in biological materials. Cryobiology, 2, $165-170$.

RINFRET, A. P. 1966 a Thermal history. Cryobiology, 2, 171-180.

RiNfRET, A. P. 1966 b Concluding notes on the symposium on freezing rates. Cryobiology, 2, 206209.

RowE, A. W. 1966 Biochemical aspects of cryoprotective agents in freezing and thawing. Cryobio$\log y, 3,12-18$.

Rowé, A. W. and RinfRet, A. P. 1962 Controlled rate freezing of bone marrow. Blood, 20, 636.

RowE, A. W. and FELLIG, J. 1963 Evaluation of liquid nitrogen preserved bone marrow cells by metabolic techniques. Blood, 21, 739 .

SmrTh, A. U. 1961 Biological Effects of Freezing and Supercooling. Williams and Wilkins Co., Baltimore, Md, $440 \mathrm{pp}$.

TILl, J. E. and MCCullough, E. A. 1961 A direct measurement of the radiation sensitivity of. normal mouse bone marrow cells. Radiat. Res., 14, 213-222. 